\title{
Transcriptome analysis of Sporisorium scitamineum reveals critical environmental signals for fungal sexual mating and filamentous growth
}

\author{
Meixin Yan ${ }^{1,3}$, Weijun Dai ${ }^{2,3}$, Enping Cai ${ }^{2,3}$, Yi Zhen Deng ${ }^{1,3}$, Changqing Chang ${ }^{2,3}$, Zide Jiang ${ }^{1 *}$
} and Lian-Hui Zhang ${ }^{1,3^{*}}$

\begin{abstract}
Background: Sporisorium scitamineum causes the sugarcane smut disease, one of the most serious constraints to global sugarcane production. S. scitamineum possesses a sexual mating system composed of two mating-type loci, $a$ and $b$ locus. We previously identified and deleted the $b$ locus in S. scitamineum, and found that the resultant Ss $\triangle$ MAT-1b mutant was defective in mating and pathogenicity.

Results: To further understand the function of b-mating locus, we carried out transcriptome analysis by comparing the transcripts of the mutant strain Ss $\triangle M A T-16$, from which the SsbE1 and SsbW1 homeodomain transcription factors have previously been deleted, with those from the wild-type MAT-1 strain. Also the transcripts from Ss $\triangle M A T-16 \times$ MAT-2 were compared with those from wild-type MAT-1 X MAT-2 mating. A total of 209 genes were up-regulated $(p<0.05)$ in the Ss $\triangle M A T-16$ mutant, compared to the wild-type MAT-1 strain, while 148 genes down-regulated $(p<0.05)$. In the mixture, 120 genes were up-regulated $(p<0.05)$ in Ss $\triangle M A T-1 b$ X MAT-2, which failed to mate, compared to the wild-type MAT-1 X MAT-2 mating, and 271 genes down-regulated $(p<0.05)$. By comparing the up- and down-regulated genes in these two sets, it was found that 15 up-regulated and 37 down-regulated genes were common in non-mating haploid and mating mixture, which indeed could be genes regulated by b-locus. Furthermore, GO and KEGG enrichment analysis suggested that carbon metabolism pathway and stress response mediated by Hog1 MAPK signaling pathway were altered in the non-mating sets.
\end{abstract}

Conclusions: Experimental validation results indicate that the bE/bW heterodimeric transcriptional factor, encoded by the $b$-locus, could regulate $S$. scitamineum sexual mating and/or filamentous growth via modulating glucose metabolism and Hog1-mediating oxidative response.

Keywords: Mating, Sugarcane smut, bE/bW heterodimeric transcriptional factor, Glucose, Hog 1

\section{Background}

Sugarcane smut is a devastating disease in sugarcane growing areas globally. The characteristic symptom of the disease is a black or gray growth that is referred to as a "smut whip" [1]. Sugarcane smut is caused by the fungus S. scitamineum, a bipolar species $[2,3]$ with two

\footnotetext{
*Correspondence: zdjiang@scau.edu.cn; Ihzhang01@scau.edu.cn Meixin Yan and Weijun Dai are co-first authors.

${ }^{1}$ Guangdong Province Key Laboratory of Microbial Signals and Disease Control, College of Agriculture, South China Agricultural University, Guangzhou, Peoples' Republic of China

Full list of author information is available at the end of the article
}

mating type strains MAT-1 and MAT-2 [4] producing haploid sporidia by budding. The compatible sporidia fuse to develop pathogenic dikaryotic hyphae, which grow within the stalk of sugarcane and form diploid teliospores to complete the pathogenic life cycle [3]. The teliospores are disseminated by wind or rain splashes and germinate to form four sporidia, and initiate next round of life cycle by mating. The sexual mating process of S. scitamineum is similar to the maize pathogen Ustilago maydis, which is regulated by two unlinked mating type loci, $a$ locus and $b$ locus [5-7]. The bi-allelic $a$ loci that 
encode a pheromone/pheromone receptor system that is responsible for recognition of the opposite haploid sporidia and formation of conjugation tubes [8]. The $b$ locus composed of the $b E$ and $b W$ genes, encoding a heterodimeric transcription factor to maintain the dikaryotic filament and promote subsequent penetration of the host plant, after fusion of the sporidia [8-10].

It has been reported that in $U$. maydis, the $\mathrm{bE} / \mathrm{bW}$ transcription factor acts through a regulatory cascade to affect various pathways in triggering pathogenic development, including cell cycle regulation, mitosis and DNA replication [11]. However, the physiology of S. scitamineum mating is largely unknown, due to unavailability of genome sequence and effective method of genetic manipulation, previously. Recently, with the genome sequencing performed by Que et al. [2] and Taniguti et al. [12], and optimizing of the ATMT transformation procedure for S. scitamineum [13], investigation on $S$. scitamineum differentiation and pathogenesis on molecular level becomes feasible. Recently, we identified and characterized a b-locus homolog in S. scitamineum, and found that it is essential for sexual mating and filamentous growth [14], but the underlying mechanism remained unclear. Given that b-locus encodes a homeodomain transcription complex, comparative transcriptome analysis may provide useful clues to possible b-locus target gene(s) and functional study of such candidate gene(s) may reveal the molecular basis of b-locus regulating S. scitamineum sexual mating and/or filamentous growth. Therefore, we carried out transcriptome analysis with wild-type $M A T-1$ and $S s \triangle M A T-1 b$ mutant, and with mating and non-mating mixtures of S. scitamineum haploids. Our study identified several potential target genes of b-locus encoding transcriptional factor, that are likely involved in S. scitamineum sexual mating and/or filamentous growth, and further reveals two critical endogenous/environmental cues: nutrient and redox homeostasis, for mating and/or filementous growth in S. scitamineum.

\section{Methods}

\section{Growth conditions and strains used in this study}

Teliospores of sugarcane smut were collected from the fields in Guangdong province of China $\left(21^{\circ} 12^{\prime} 36^{\prime \prime} \mathrm{N}\right.$; $\left.101^{\circ} 10^{\prime} 12^{\prime \prime} \mathrm{E}\right)$, and no specific permissions were required for sampling diseased plants in this location. Haploid colonies of $M A T-1$ and $M A T-2$ were isolated from these teliospores by serial dilution and plating on YePSA medium, as previously described [15]. Synthetic complete dextrose (SCD) medium is consisted of $0.7 \%$ (wt/vol) yeast nitrogen base without amino acids, $0.17 \%$ complete amino acids powder, and $2 \%$ (wt/vol) glucose [16]. Synthetic complete (SC) medium was formulated as SCD medium without addition of glucose [16].

\section{RNA extraction and sequencing strategies}

TRIzol Reagent (Life Technologies, UK) was used for Total RNA extraction from haploid $M A T-1$ and $S s \triangle M A T$ $1 b$ mutant. MAT-1 and MAT-2 haploids were mixed and plated on YePSA medium for $24 \mathrm{~h}$ before total RNA extraction with TRIzol Reagent. Similarly, Ss $\triangle M A T-1 b$ and $M A T-2$ haploids were mixed and inoculated on YePSA medium for $24 \mathrm{~h}$ before total RNA extraction.

Libraries were constructed following Illumina manufacturer's protocol of the "TruSeq RNA Sample Prep v2 Low Throughput (LT)" kit. Paired-end sequencing was performed on the Illumina $\mathrm{HiSeq}^{\mathrm{TM}} 2000$. Reads were analyzed by FASTQC (http://www.bioinformatics.babraham.ac.uk/projects/fastqc/) and low quality bases (phred $\geq 20$ ), Illumina adapters and poly-A tails were removed using the NGS QC Toolkit v2.3.3 (http://59.163.192.90:8080/ngsqctoolkit/) [17].

\section{Transcriptome assembly and annotations}

De novo short read assembly was performed using tophat and cufflinks softwares [18]. The assembled reads were mapped to the complete genome of S. scitamineum SSC39B strain (ftp://ftp.ncbi.nlm.nih.gov/genomes/genbank/fungi/Sporisorium_scitamineum/latest_assembly_versions/GCA_000772675.1_Sporisorium_scitamineum_v1) using Tophat and Bowtie2 [19].

Unigene generated by De novo short read assembly was aligned to NCBI NR Database (ftp://ftp.ncbi. nih.gov/blast/db), SWISSPROT Database (http://www. uniprot.org/downloads), and KOG Database (Clusters of orthologous groups for eukaryotic complete genomes, ftp://ftp.ncbi.nih.gov/pub/COG/KOG/kyva), respectively. Unigene encoding proteins with high similarity $(\mathrm{e}<1 \mathrm{e}-5)$ to the known proteins in aforementioned databases were used to annotate the corresponding Unigene. GO annotation was performed by Blast2GO software [20] and the database http://www.geneontology.org/. KEGG annotation was performed with the database http://www.genome.jp/kegg/pathway.html [21].

\section{Transcriptome analysis}

Differential transcript accumulation among treatments (Ss $\triangle M A T-1 b$ vs $M A T 1$, Ss $\triangle M A T-1 b$ X MAT-2 vs $M A T-1$ $\mathrm{X} M A T-2)$ was observed using bowtie2 (http://bowtiebio.sourceforge. net/bowtie2/manual.shtml) [19] and eXpress [22]. The gene expression level is calculated by using FPKM method (fragments Per kb per Million reads) [22]. Baggerley's test and the false discovery rate (FDR) with a significance level of $\leq 0.05$ and the absolute value of Log2Ratio $\geq 1$ was set as the threshold to judge the significance of gene expression difference.

GO enrichment analysis was performed as firstly mapping all DEGs (Differential Expressed Genes) to GO terms in the database (http://www.geneontology.org/), 
calculating gene numbers for every term, then using hypergeometric test to find significantly enriched GO terms in the input list of DEGs, based on GO::TermFinder (http://smd.stanford.edu/help/GOTermFinder/GO_Term Finder_help.shtml/). $P$ value was calculated using the following formula:

$$
P=1-\sum_{i=0}^{m-1} \frac{\left(\begin{array}{c}
M \\
i
\end{array}\right)\left(\begin{array}{c}
N-M \\
n-i
\end{array}\right)}{\left(\begin{array}{c}
N \\
n
\end{array}\right)}
$$

Where $\mathrm{N}$ is the number of all genes with GO annotation; $\mathrm{n}$ is the number of DEGs in $\mathrm{N}$; $\mathrm{M}$ is the number of all genes that are annotated to certain GO terms; $m$ is the number of DEGs in $\mathrm{M}$. The calculated $\mathrm{p}$-value goes through Bonferroni Correction [23], taking corrected $p$-value $\leq 0.05$ as a threshold. GO score was calculated as follows: Enrichmentscore $=\frac{m}{n} / \frac{M}{N}$.

KEGG database is used to perform pathway enrichment analysis of DEGs. The calculating formula is the same as that in GO analysis. Here $\mathrm{N}$ is the number of all genes that with KEGG annotation, $\mathrm{n}$ is the number of DEGs in N, M is the number of all genes annotated to specific pathways, and $\mathrm{m}$ is the number of DEGs in $\mathrm{M}$.

\section{Results}

\section{Unigenes identification and gene annotation}

Our RNAseq analysis produced a total length of 17.8344 Mb (Table 1) for all the transcripts, out of $2 \mathrm{G}$ clean sequencing data, representing about $100 \mathrm{X}$ coverage of the transcriptome. Compared to previous published genomic sequence of $S$. scitamineum $[2,12]$, the total length of sequence is slightly low, likely due to the fact that only transcripts (with poly-A tails) were anchored and sequenced in this study. De novo assembly of transcripts was performed as described in Methods. We identified 7341 unigenes in total, with length from 145 bp to 16628 bp (Table 1). Most of the identified unigenes are of 200-2000 bp (Fig. 1a), and GC content is within the range of 50-60\% (Fig. 1b). The unigenes were mapped to NR, SWISSPROT, and KOG Database for annotation, as listed in Additional file 1: Table S1.
Transcriptome analysis identified differentially expressed genes between mating and non-mating strains/conditions In this study, we compared two sets of non-mating vs mating strain/condition, with an aim to identify the genes related to $S$. scitamineum mating and likely regulated by $b$-locus. Differentially Expressed Genes (DEGs) were identified in the $S s \triangle M A T-1 b$ mutant compared to the wild-type $M A T-1$ strain, as well as in the non-mating mixture, Ss $\triangle M A T-1 b X M A T-2$, compared to the mating mixture of $M A T-1$ X MAT-2. DEGs of significance $(p \leq 0.05)$ in the haploid and mating set were listed in Additional file 2: Table S2 and Additional file 3: Table S3 respectively. In total, there are 357 DEGs identified in the Ss $\triangle M A T-1 b$ mutant, among which 209 genes were up-regulated and 148 down-regulated (Table 2). Under mating condition, a total of 391 genes were differentially expressed in the non-mating mixture, with 120 upregulated and 271 down-regulated (Table 2). By comparing the up- and down-regulated genes in these two conditions, we found that 15 up-regulated and 37 down-regulated genes were common in non-mating haploid and mating mixture. We listed in Table 3 for those with annotation in SWISSPROT Database.

Among the 12 up-regulated and 16 down-regulated genes listed in Table 3, we noticed that genes encoding components of signaling pathway, e.g. MAPK Cek1 (involved in mitosis in yeast [24] and fungicidal activity in Candida albicans [25]) and Hog1 (oxidative or osmotic stress response [26-28]), GTPase-activating protein BEM2/IPL2 (for cellular morphogenesis and interacting with mitosis regulator in yeast [29]), or histidine kinase (possibly involved in two-component signal pathway [30]) were up-regulated with deletion of b-locus. Also, proteins involved vesicular trafficking (AP-3 complex subunit) or metal-nicotianamine transporter YSL2 were possibly repressed by b-locus transcriptional factor (Table 3). Another transcriptional factor, RFX4, and an RNA helicase were potentially repressed by b-locus too (Table 3 ). This result indicates that b-locus may negatively regulate some signaling pathway and repressed transcription of a set of downstream genes, directly or indirectly, after sexual mating induced and during filamentous growth. On the other hand, genes induced, directly or indirectly, by b-locus include several other transcriptional factors, e.g. ATHB-54 [31], MKL/myocardin-like protein [32], Short

Table 1 Unigene statistics

\begin{tabular}{lllllllll}
\hline & All & $>=200 \mathrm{bp}$ & $>=500 \mathrm{bp}$ & $>=1000 \mathrm{bp}$ & Total Length (Mb) & Max Length & Min Length & Avg Length \\
\hline PRJNA240344 & - & - & - & - & 19.7235 & - & - & - \\
PRJEB5169 & 7711 & - & - & - & 19.4279 & - & - & - \\
PRJNA275631 & 6677 & - & - & - & 20.0676 & - & - \\
Unigene & 7341 & 7338 & 7131 & 6123 & 17.8344 & 16628 & 145 & 2429.42 \\
\hline
\end{tabular}




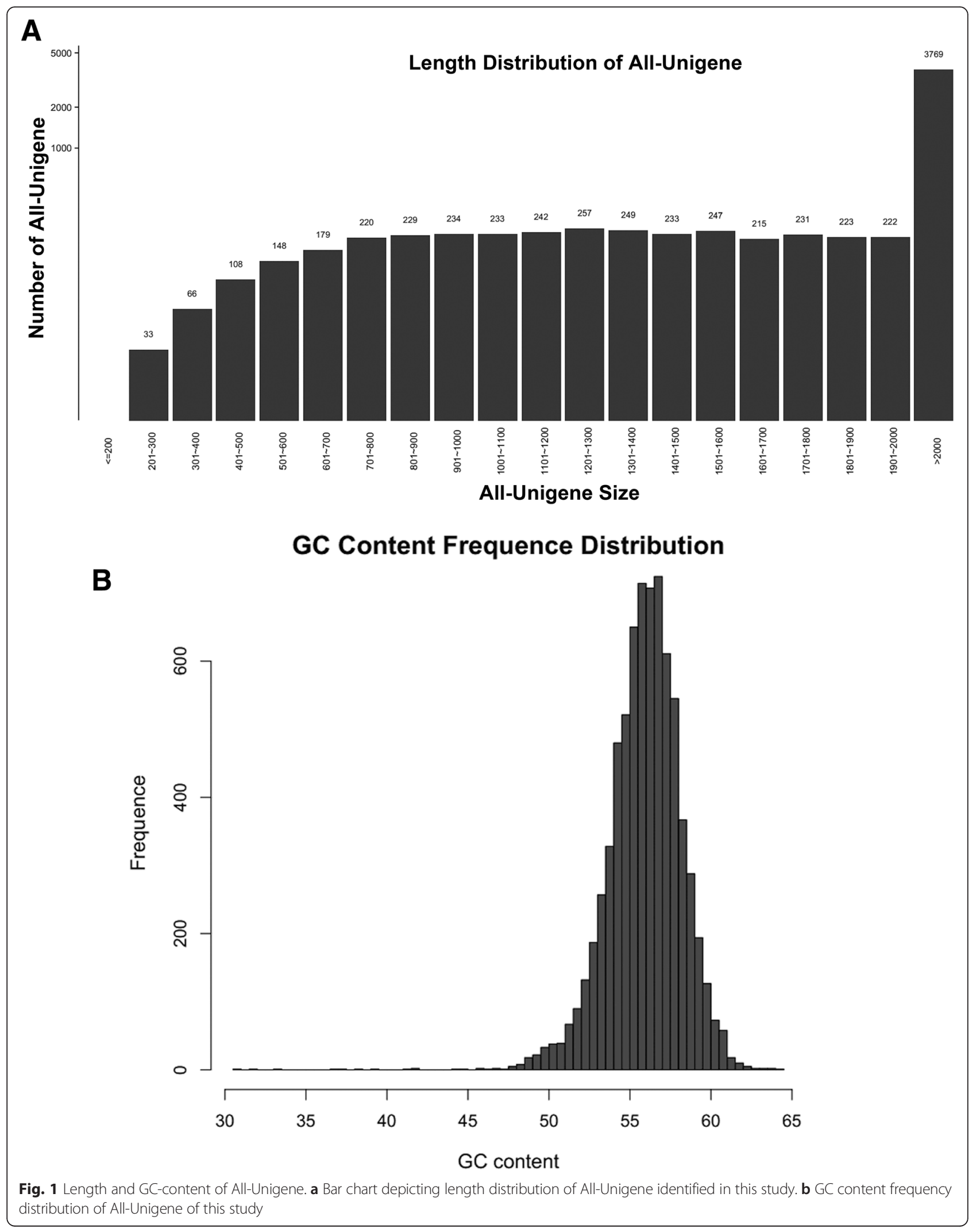


Table 2 DEGs statistics

\begin{tabular}{lllll}
\hline Control & Case & Up_diff & Down_diff & Total_diff \\
\hline MAT-1 & b-deletion & 209 & 148 & 357 \\
MAT-1 b-deletion + MAT-2 & MAT-1 + MAT-2 & 120 & 271 & 391
\end{tabular}

stature homeobox protein 2 (Shox2; related to growth and development in human [33]; Table 3). b-locus may also induce regulators involved in biosynthesis, including polyol transporter 5, Rhamnolipids biosynthesis 3-oxoacyl-[acylcarrier-protein] reductase, MFS-type transporter, and several hydrolases or Glucoamylase, during mating and/or filamentous growth (Table 3). PKA and MAPK signaling pathway were found to be involved in b-locus regulating sexual mating and/or filamentous growth in $U$. maydis [11]. Here in our study, we also identified component of MAPK pathway, Cek1 and Hog1, that was potentially regulated by $S$. scitamineum b-locus, but not among those identified in $U$. maydis. Our finding indicates that $S$. scitamineum b-locus may regulate small molecular (e.g. metal-nicotianamine, polyol) transport, vesicular trafficking, biosynthesis, stress-response mediated by MAPK signaling (Hog1), and a cascade of transcriptional network, during mating and/or filamentous growth. The candidate genes listed in Table 3 are of great interest in our future investigation, in terms of elucidating physiology and molecular mechanism of S. scitamineum differentiation and pathogenesis.

\section{Identification of starch/sucrose metabolism and Hog1 MAPK pathway in fungal mating}

As an international standard gene functional classification system, Gene Ontology (GO), offers a dynamic-updated controlled vocabulary, as well as a strictly defined concept to comprehensively describe properties of genes and their products in any organism [34]. Therefore GO enrichment analysis of the aforementioned DEGs may further reveal the functional relevance of $b$-locus regulating genes and $S$. scitamineum mating. Enriched GO (for both up- and down- regulated) in the haploid and mating sets were listed in Additional file 4: Table S4 and Additional file 5: Table S5 respectively, and schematically represented following three ontologies (molecular function, cellular component and biological process) as in Fig. 2. Among them, we noticed that the genes involved in membrane transport, oxidation-reduction process and ATP-binding were overall differentially regulated in non-mating haploid (Ss $\triangle M A T-1 b$ mutant), as well as in non-mating mixture (Ss $\triangle M A T-1 b X M A T-2$, Fig. 2). However, some particular genes associated with the membrane transport process (GO: 0055085) were up-regulated, while some others, enriched in the same GO term, were down-regulated, in both non-mating haploid and non-mating mixture (Additional file 4: Table S4 and Additional file 5: Table S5).
Similar situation occurred for oxidation-reduction process (GO: 0055114; Additional file 4: Table S4 and Additional file 5: Table S5) as well as ATP-binding (GO: 0005524; Additional file 4: Table S4 and Additional file 5: Table S5). On the other hand, ATP catabolic process (GO: 0006200) was up-regulated in both $S s \triangle M A T-1 b \mathrm{mu}-$ tant and $S s \triangle M A T-1 b X M A T-2$ mixture (Additional file 4: Table S4 and Additional file 5: Table S5), indicating that S. scitamineum mating may repress ATP catabolism. In summary, GO terms enrichment analysis further verifies that metabolism, biosynthsis, transmembrane transport and redox homeostasis would be tightly regulated by b-locus during $S$. scitamineum mating and/or filamentous growth.

Genes usually interact with each other to play roles in certain biological functions. Pathway-based analysis helps to further understand the biological functions of unigenes. KEGG-enrichment analysis thus was carried out to identify significantly enriched metabolic pathways or signal transduction pathways in DEGs comparing with the whole genome background [21]. Enriched KEGG terms were listed in Additional file 6: Table S6 and Additional file 7: Table S7, for $S s \triangle M A T-1 b$ vs wildtype $M A T-1$ and the non-mating mixture of $S s \triangle M A T-1 b$ X $M A T-2$ vs wild-tyype $M A T-1$ X $M A T-2$, respectively. Among the enriched pathways, we observed that starch and sucrose metabolism pathway (ko00500; Additional file 8: Figure S1) was commonly found in both haploid and mating sets. The predicted outcome of differentially regulation of this pathway was that glucose production would be reduced, while accumulation of $1,3-\beta$-glucan would be increased (Additional file 8: Figure S1), in Ss $\triangle M A T-1 b$ or non-mating mixture. Another commonly up-regulated gene, Hog1 (p38), was also found in enriched KEGG pathway (ko04010, MAPK signaling) in both $S s \triangle M A T-1 b$ or non-mating mixture (Additional file 9: Figure S2). Hog1 mediates osmo- and oxidative stress response in yeast and fungi [26-28], and is important for mating capacity in Candida albicans [16]. We infer that carbohydrate metabolism as well as redox homeostasis may play important roles in S. scitamineum mating, and be subjective to regulation (directly or indirectly) by the $b$-locus.

\section{Starch/sucrose metabolism and Hog1 MAPK pathway may regulate $S$. scitamineum mating}

To verify the involvement of starch/sucrose metabolism and Hog1 MAPK pathway in S. scitamineum mating, we tested the growth of the wild-type MAT-1, MAT-2 and Ss $\triangle M A T-1 b$ mutant, as well as mating MAT-1 X MAT-2 mixtures, under osmotic and oxidative stresses. The results showed that $S s \triangle M A T-1 b$ was more resistant to oxidative stress, compared to the wild-type $M A T-1$ as well as mating mixture (Fig. 3a middle panel). However, wild- 
Table 3 List of Up- and Down- regulated genes common in Ss $\triangle M A T-16$ vs wild-type MAT-1 and Ss $\triangle M A T-16$ X MAT-2 vs wild-type MAT-1 X MAT-2 comparing sets

\begin{tabular}{|c|c|c|c|c|c|c|}
\hline \multirow[t]{2}{*}{ Swiss ID } & \multirow[t]{2}{*}{$\begin{array}{l}\text { Length } \\
\text { (bp) }\end{array}$} & \multirow{2}{*}{$\begin{array}{l}\text { Fold } \\
\text { change } \\
\text { Ss } \triangle M A T-16 \\
\text { vs MAT-1 }\end{array}$} & \multirow[t]{2}{*}{$p$ value } & \multirow{2}{*}{$\begin{array}{l}\text { Fold change } \\
\text { Ss } \triangle M A T-1 b \times \\
\text { MAT-2 vs MAT-1 } \\
\text { X MAT-2 }\end{array}$} & \multirow[t]{2}{*}{$p$ value } & \multirow[t]{2}{*}{ swiss.Description } \\
\hline & & & & & & \\
\hline sp|P38938|CEK1_SCHPO & 13208 & 13.42056 & $1.68 \mathrm{E}-06$ & 3.44586 & 0.01867 & $\begin{array}{l}\text { Serine/threonine-protein kinase cek1 OS }=\text { Schizosaccharomyces } \\
\text { pombe (strain 972/ATCC 24843) GN }=\text { cek1 PE }=1 \mathrm{SV}=3\end{array}$ \\
\hline sp|P39960|BEM2_YEAST & 8330 & 1.796835 & 0.023621 & 1.718937 & 0.023855 & $\begin{array}{l}\text { GTPase-activating protein BEM2/IPL2 OS = Saccharomyces } \\
\text { cerevisiae (strain ATCC 204508/S288c) GN = BEM2 PE = } 1 \mathrm{SV}=1\end{array}$ \\
\hline sp|Q6R3K9|YSL2_ARATH & 2834 & 1.946333 & 0.003032 & 1.913564 & 0.010979 & $\begin{array}{l}\text { Metal-nicotianamine transporter YSL2 OS = Arabidopsis } \\
\text { thaliana GN }=\text { YSL2 PE }=2 S V=1\end{array}$ \\
\hline sp|Q9JME5|AP3B2_MOUSE & 9407 & 12.37208 & 9.87E-06 & 4.222222 & $2.16 \mathrm{E}-05$ & $\begin{array}{l}\text { AP-3 complex subunit beta- } 2 \mathrm{OS}=\text { Mus musculus } \mathrm{GN}=\mathrm{Ap} 3 \mathrm{~b} 2 \\
\mathrm{PE}=1 \mathrm{SV}=2\end{array}$ \\
\hline sp|Q12019|MDN1_YEAST & 16628 & 1.87115 & 0.005704 & 2.001882 & 0.002886 & $\begin{array}{l}\text { Midasin OS = Saccharomyces cerevisiae (strain ATCC 204508/ } \\
\text { S288C) GN = MDN1 PE }=1 \mathrm{SV}=1\end{array}$ \\
\hline sp|Q54YH4|DHKB_DICDI & 6828 & $\operatorname{lnf}$ & 0.007605 & 10.75 & 0.005969 & $\begin{array}{l}\text { Hybrid signal transduction histidine kinase } \mathrm{B} O S=\text { Dictyostelium } \\
\text { discoideum } \mathrm{GN}=\mathrm{dhkB} P E=1 \mathrm{SV}=1\end{array}$ \\
\hline sp|A2BGA0|RFX4_DANRE & 4929 & 6.911589 & $1.76 \mathrm{E}-06$ & 1.937224 & 0.015736 & Transcription factor RFX4 OS = Danio rerio GN $=r f \times 4 \mathrm{PE}=2 \mathrm{SV}=1$ \\
\hline sp|Q4P3W3|DBP10_USTMA & 3384 & 12.41402 & 0.025351 & 4.851852 & 0.046387 & $\begin{array}{l}\text { ATP-dependent RNA helicase DBP10 OS = Ustilago maydis } \\
\text { (strain 521/FGSC 9021) GN = DBP10 PE }=3 \mathrm{SV}=1\end{array}$ \\
\hline sp|P56584|SID1_USTMA & 2399 & 1.764062 & 0.017173 & 2.524876 & 0.016416 & $\begin{array}{l}\text { L-ornithine 5-monooxygenase OS = Ustilago maydis } \\
\text { (strain 521/FGSC 9021) GN }=\mathrm{SID} 1 \mathrm{PE}=2 \mathrm{SV}=2\end{array}$ \\
\hline sp|P36619|PMD1_SCHPO & 7057 & 2.328467 & 0.01718 & 2.990596 & 0.006957 & $\begin{array}{l}\text { Leptomycin B resistance protein pmd1 OS }=\text { Schizosaccharomyces } \\
\text { pombe (strain 972/ATCC 24843) } \mathrm{GN}=\text { pmd1 } \mathrm{PE}=3 \mathrm{SV}=2\end{array}$ \\
\hline sp|Q4PC06|HOG1_USTMA & 5538 & 3.954272 & 0.001332 & 2.294118 & 0.037876 & $\begin{array}{l}\text { Mitogen-activated protein kinase HOG1 OS = Ustilago } \\
\text { maydis (strain 521/FGSC 9021) GN = HOG1 PE = } 3 \mathrm{SV}=1\end{array}$ \\
\hline sp|P0CJ65|ATB54_ARATH & 2265 & 0.02171 & $6.20 \mathrm{E}-19$ & 0.178523 & $3.40 \mathrm{E}-06$ & $\begin{array}{l}\text { Homeobox-leucine zipper protein ATHB-54 OS = Arabidopsis } \\
\text { thaliana GN }=\text { ATHB-54 PE }=2 \mathrm{SV}=1\end{array}$ \\
\hline sp|P22943|HSP12_YEAST & 1053 & 0.168388 & $9.19 \mathrm{E}-15$ & 0.552964 & 0.01578 & $\begin{array}{l}12 \mathrm{kDa} \text { heat shock protein OS = Saccharomyces cerevisiae } \\
\text { (strain ATCC 204508/S288c) GN }=\mathrm{HSP} 12 \mathrm{PE}=1 \mathrm{SV}=1\end{array}$ \\
\hline sp|O14094|PPX1_SCHPO & 2702 & 0.36517 & 0.002301 & 0.144718 & 0.000202 & $\begin{array}{l}\text { Putative exopolyphosphatase OS = Schizosaccharomyces pombe } \\
\text { (strain 972/ATCC 24843) GN }=\text { SPAC2F3.11 PE }=3 \mathrm{SV}=1\end{array}$ \\
\hline sp|Q6CHP9|CCM1_YARLI & 2835 & 0.451759 & 0.010845 & 0.049724 & $1.68 \mathrm{E}-07$ & $\begin{array}{l}\text { Mitochondrial group I intron splicing factor CCM1 OS = Yarrowia } \\
\text { lipolytica (strain CLIB 122/E 150) GN =CCM1 PE }=3 \mathrm{SV}=1\end{array}$ \\
\hline sp|P22018|B4_USTMD & 1539 & 0 & $9.33 \mathrm{E}-23$ & 0.325834 & 0.001866 & $\begin{array}{l}\text { Mating-type locus allele B4 protein OS = Ustilago maydis } \\
\mathrm{PE}=3 \mathrm{SV}=1\end{array}$ \\
\hline sp|Q8VZ80|PLT5_ARATH & 2427 & 0.452944 & 0.022087 & 0.493914 & 0.003544 & $\begin{array}{l}\text { Polyol transporter } 5 \mathrm{OS}=\text { Arabidopsis thaliana } \mathrm{GN}=\mathrm{PLT5} \\
\mathrm{PE}=1 \mathrm{SV}=2\end{array}$ \\
\hline sp|Q4WFX9|LAP2_ASPFU & 2169 & 0.053682 & 0.00954 & 0.337114 & 4.19E-05 & $\begin{array}{l}\text { Probable leucine aminopeptidase } 2 \text { OS = Neosartorya } \\
\text { fumigata (strain ATCC MYA-4609/Af293/CBS 101355/ } \\
\text { FGSC A1 100) GN = lap2 PE = } 3 \mathrm{SV}=2\end{array}$ \\
\hline sp|Q5UP73|YR614_MIMIV & 1884 & 0.353454 & 2.79E-06 & 0.340428 & $1.15 \mathrm{E}-05$ & $\begin{array}{l}\text { Putative band } 7 \text { family protein } \mathrm{R} 614 \mathrm{OS}=\text { Acanthamoeba } \\
\text { polyphaga mimivirus } \mathrm{GN}=\mathrm{MIMI} \_\mathrm{R} 614 \mathrm{PE}=3 \mathrm{SV}=1\end{array}$ \\
\hline sp|Q8K4J6|MKL1_MOUSE & 736 & 0.600119 & 0.019664 & 0.330712 & $5.01 \mathrm{E}-06$ & $\begin{array}{l}\mathrm{MKL} / \text { myocardin-like protein } 1 \mathrm{OS}=\text { Mus musculus } \mathrm{GN}=\mathrm{Mkl} 1 \\
\mathrm{PE}=1 \mathrm{SV}=2\end{array}$ \\
\hline sp|Q9RPT1|RHLG_PSEAE & 1073 & 0.337766 & 0.035915 & 0.16568 & 0.019132 & $\begin{array}{l}\text { Rhamnolipids biosynthesis 3-oxoacyl-[acyl-carrier-protein] } \\
\text { reductase OS = Pseudomonas aeruginosa (strain ATCC 15692/ } \\
\text { PAO1/1C/PRS 101/LMG 12228) GN = rhlG PE }=1 \mathrm{SV}=1\end{array}$ \\
\hline sp|P80299|HYES_RAT & 1160 & 0.467807 & 0.005301 & 0.425344 & 0.024971 & $\begin{array}{l}\text { Bifunctional epoxide hydrolase } 2 \mathrm{OS}=\text { Rattus norvegicus } \\
\mathrm{GN}=\mathrm{Eph} \times 2 \mathrm{PE}=1 \mathrm{SV}=1\end{array}$ \\
\hline sp|P36914|AMYG_ASPOR & 2852 & 0.569668 & 0.010942 & 0.419058 & 0.010065 & $\begin{array}{l}\text { Glucoamylase OS = Aspergillus oryzae (strain ATCC 42149/RIB 40) } \\
\text { GN = glaA PE }=2 \mathrm{SV}=2\end{array}$ \\
\hline sp|P34211|YUAR_ECOLI & 2743 & 0.226761 & $3.58 \mathrm{E}-06$ & 0.318024 & $3.27 \mathrm{E}-05$ & $\begin{array}{l}\text { Putative hydrolase YuaR OS = Escherichia coli (strain K12) } \\
\mathrm{GN}=\text { yuaR PE }=3 \mathrm{SV}=3\end{array}$ \\
\hline
\end{tabular}


Table 3 List of Up- and Down- regulated genes common in Ss $\triangle M A T-16$ vs wild-type MAT-1 and Ss $\triangle M A T-16$ X MAT-2 vs wild-type MAT-1 X MAT-2 comparing sets (Continued)

\begin{tabular}{|c|c|c|c|c|c|c|}
\hline sp|O35750|SHOX2_RAT & 3616 & 0.490521 & 0.005405 & 0.55 & 0.023074 & $\begin{array}{l}\text { Short stature homeobox protein } 2 \text { (Fragment) OS = Rattus } \\
\text { norvegicus } \mathrm{GN}=\text { Shox } \mathrm{PE}=2 \mathrm{SV}=2\end{array}$ \\
\hline sp|Q767C8||H5GT_IRIHO & 2482 & 0.263235 & 3.53E-09 & 0.630828 & 0.048192 & $\begin{array}{l}\text { Cyanidin 3-O-rutinoside 5-O-glucosyltransferase OS = Iris } \\
\text { hollandica } \mathrm{GN}=5 \mathrm{GT} P E=1 \mathrm{SV}=1\end{array}$ \\
\hline sp|O42922|YBIH_SCHPO & 4584 & 0.240982 & $5.71 \mathrm{E}-10$ & 0.61912 & 0.037253 & $\begin{array}{l}\text { Uncharacterized MFS-type transporter C16A3.17c } \\
\text { OS }=\text { Schizosaccharomyces pombe (strain } 972 / \text { ATCC 24843) } \\
\text { GN }=\text { SPBC16A3.17C PE }=3 \text { SV }=1\end{array}$ \\
\hline
\end{tabular}

type $M A T-2$ also showed higher resistance to $\mathrm{H} 2 \mathrm{O} 2$ when cultured alone but not in mating condition (Fig. 3a middle panel). Osmotic stress imposed by $500 \mathrm{mM} \mathrm{NaCl}$ repressed the filamentous growth in the mating mixture of MAT-1 X MAT-2 (Table 3A right panel). However, the colonial growth was indistinguishable between the wild-type $M A T-1$ and $S s \triangle M A T-1 b$ mutant strain, under the same osmotic stress (Fig. 3a right panel). On the other hand, the YePSA medium supplemented with high concentration $(10 \%, \mathrm{wt} / \mathrm{vol})$ of glucose repressed filamentous growth in the mating mixture of $M A T-1 \mathrm{X}$ $M A T-2$ (Fig. 3b). In contrast, glucose-depleted medium (SC) was more favorable for filamentous growth in mating mixture of MAT-1 X MAT-2, compared to the SCD medium containing $2 \%$ glucose (Fig. $3 c$ ). As $1,3-\beta-$ glucan is an effective anti-oxidant, the significant enhancement of Hog1 transcripts in non-mating haploid/mixture may be an indirect consequence of elevated intracellular oxidative level in non-mating S. scitamineum haploid and mixture. Furthermore, we tested the effect of antioxidant, Glutathione (GSH) on colonial and filamentous growth of haploid and mating strains. All the strains were more resistant to GSH on SC (glucose-deplete) medium compared to SCD (glucose-containing) medium (Fig. 3c). This indicates that the glucose may indeed be utilized for synthesis of anti-oxidant 1,3- $\beta$-glucan, therefore depletion

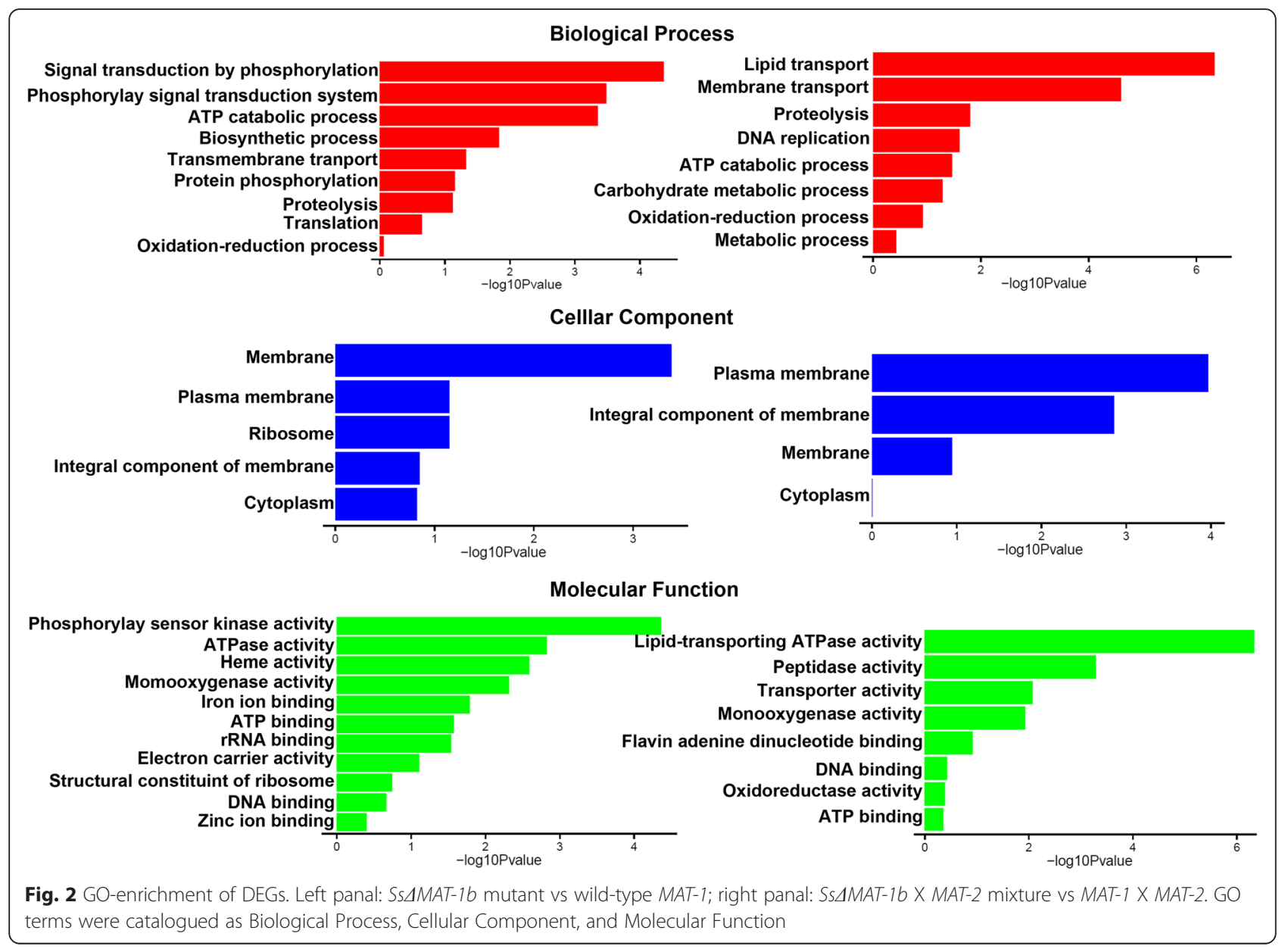




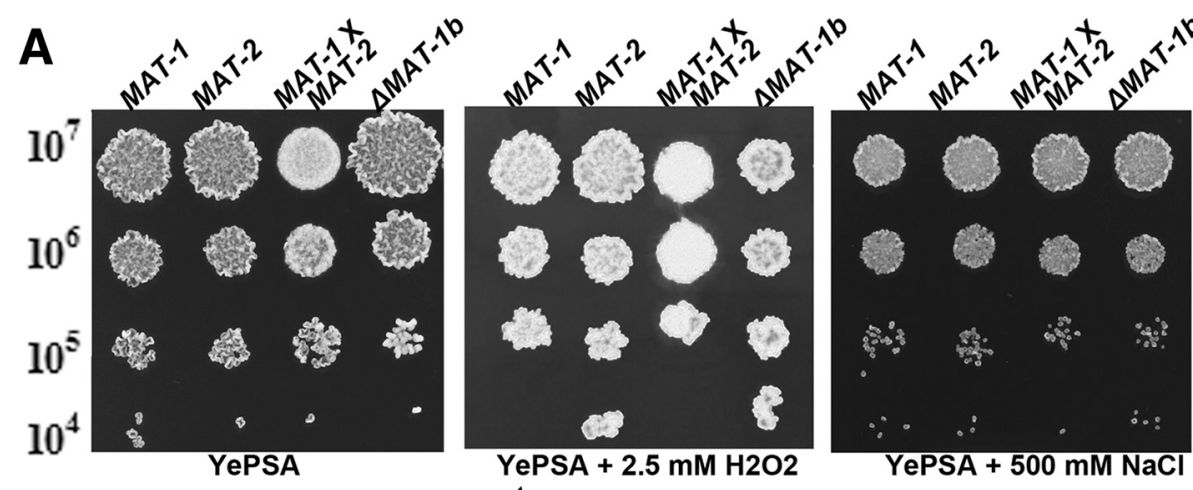

B
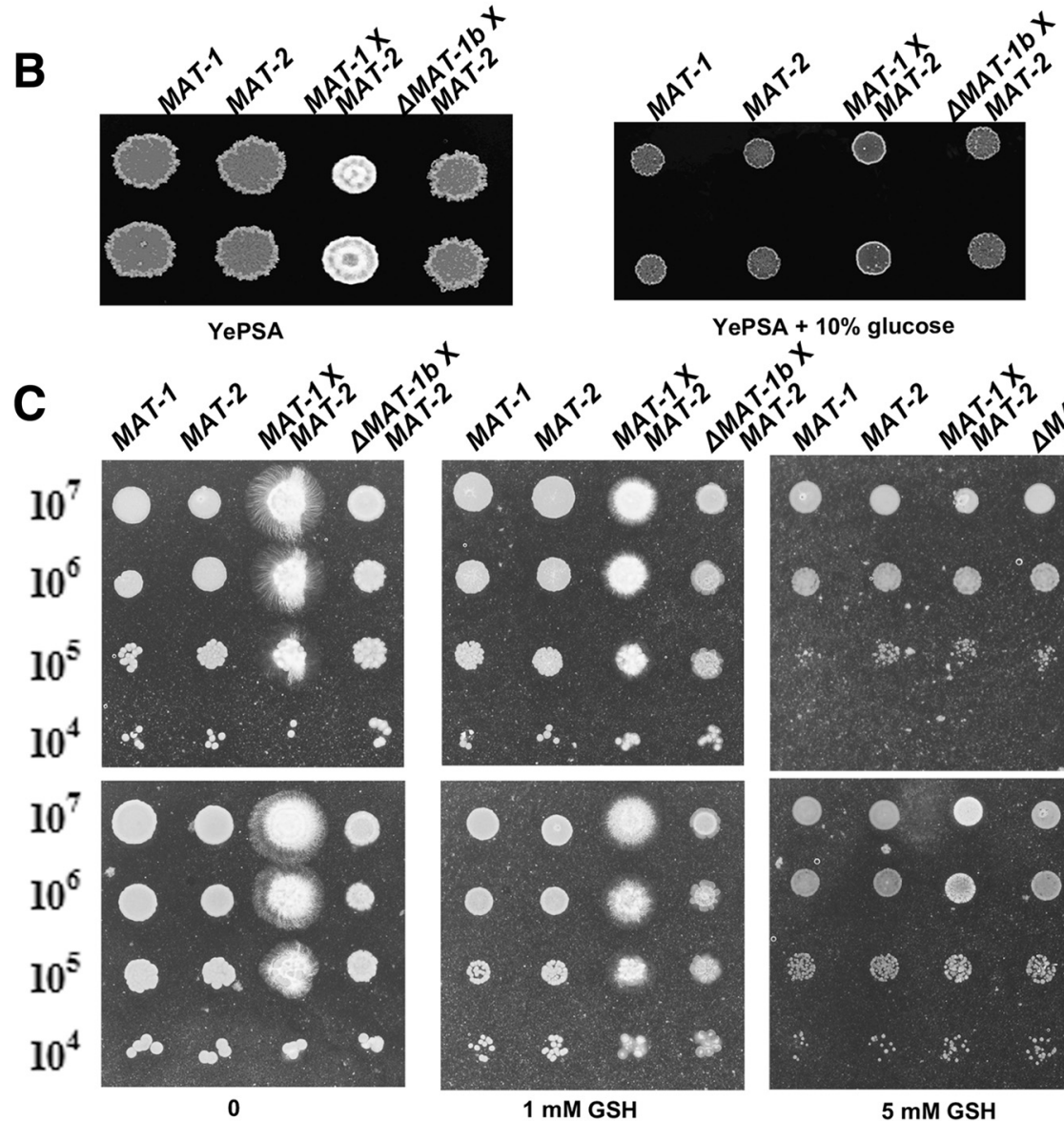

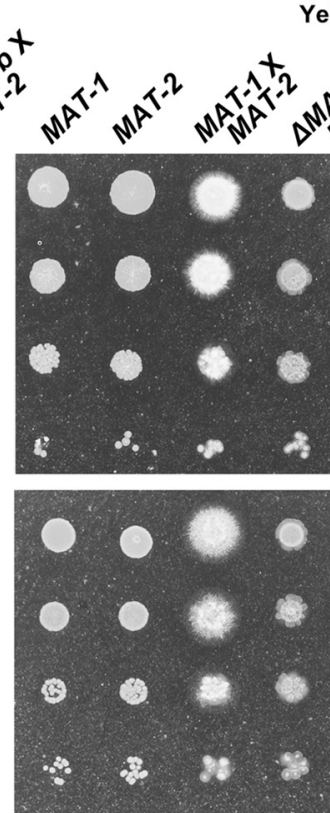

$1 \mathrm{mM} \mathrm{GSH}$
YePSA $+10 \%$ glucose
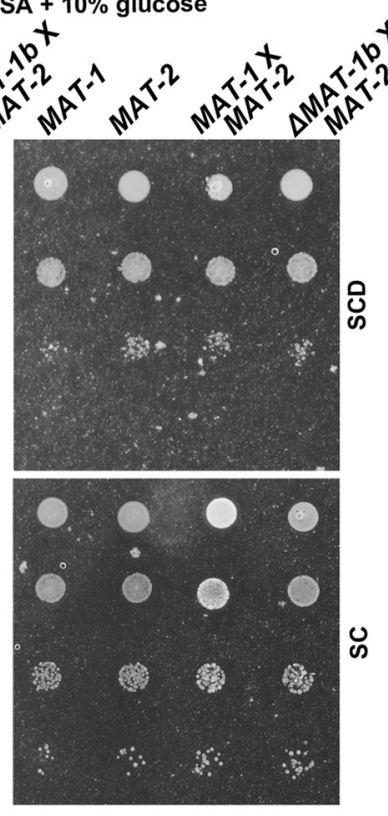

$5 \mathrm{mM} \mathrm{GSH}$

Fig. 3 Starch/sucrose metabolism and Hog1 MAPK pathway are likely involved in S. scitamineum mating, and subject to regulation of the $b$-locus. a Serially diluted cells of MAT-1, MAT-2, MAT-1 X MAT-2, and Ss $\triangle M A T-16$, were spotted onto YePSA medium supplemented with $2.5 \mathrm{mM}$ hydrogen peroxide or $500 \mathrm{mM} \mathrm{NaCl}$. b Cells of MAT-1, Ss $\triangle M A T-16$, MAT-1 X MAT-2, and Ss $\triangle M A T-1 b$ X MAT-2, were spotted onto YePSA medium with or without $10 \%(\mathrm{wt} / \mathrm{vol})$ of glucose. c Serially diluted cells of MAT-1, Ss $\triangle M A T-16$, MAT-1 X MAT-2, and SS $\triangle M A T-16$ X MAT-2, were spotted onto SCD or SC medium, with $1 \mathrm{mM}$ or $5 \mathrm{mM} \mathrm{GSH}$

of glucose resulted in more resistance to GSH, another anti-oxidant. Overall, these results indicate that glucose may play a negative role in promoting $S$. scitamineum mating and/or filamentous growth, and the $b$-locus encoding heterodimeric transcriptional factor may regulate starch/ sucrose metabolism on transcriptional level. We further predicted, based on transcriptome analysis, that the $b$-locus encoded heterodimeric transcriptional factor may regulate $S$. scitamineum mating and/or filamentous growth by promoting synthesis of $1,3-\beta$-glucan (probably from $D$ glucose) and meanwhile repressed the stress response signaling pathway mediated by Hog1 MAPK. A working model, adopted and modified from b-locus regulatory network proposed in $U$. maydis [11], is depicted in Fig. 4 . 


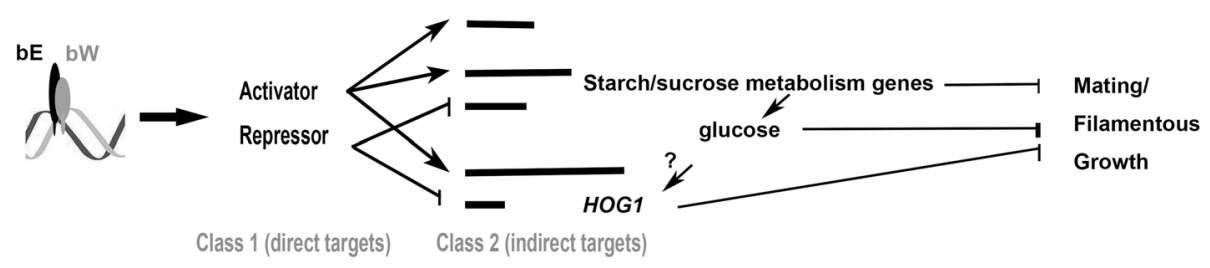

Fig. 4 Proposed Model. bE and bW proteins derived from opposite mating type form functional transcriptional complex to activate or repress Class 1 (direct) target genes. Class 2, indirect targets, are in turn activated or repressed by products encoded by Class 1 targets. Starch/sucrose metabolism genes as well as HOG1 may be indirectly repressed by the bE/bW transcriptional complex, during mating. $b$-repressed glucose metabolism gene may promote production of glucose, which may repress mating and/or filamentous growth. Meanwhile, glucose production may lead to elevated intracellular oxidative level, and thus induce Hog1 MAPK pathway, which also negatively regulates mating and/or filamentous growth. Overall, $b$-locus may act in shutting down mating/filamentous growth inhibitors, including high level of glucose and Hog1 MAPK signaling

\section{Discussion}

Investigation on molecular mechanism on S. scitamineum mating and/pathogenicity was impeded due to lack of S. scitamineum genome sequence, until 2014, when Que et al., published the first genome sequence of the pathogen [2]. More recently, a Brazil group published a complete genome assembly of S. scitamineum, as well as the fungal transcriptome profiles revealing the candidate genes unique to interaction with sugarcane [12]. Such genomic and transcriptome analyses have provided enormous convenience for functional study of mating and pathogenic genes in S. scitamineum. In current study, we conducted transcriptome analysis and comparison between mating vs non-mating haploid/mixture, which present useful information on the $b$-regulated gene expression cascade during S. scitamineum mating and/or filamentous growth. Our transcriptome analysis predicted 7341 unigenes (transcripts), which is similar to the predicted genome sizes of the three published S. scitamineum strains (http://www.ncbi.nlm.nih.gov/assembly/organism/49012/all/; Table 1). The GCcontent of our identified unigenes is ranged from 50 to $60 \%$, peaking at $55 \%$ (Fig. 1b), which is also consistent with the GC-content of these three genome projects (54.9, 54.8 and $55.04 \%$ respectively). These data suggest that our de novo assembly of transcripts in S. scitamineum is valid for the identification of DEGs as well as GO and KEGG enrichment.

Our transcriptome analyses identified 357 DEGs in Ss $\triangle M A T-1 b$ mutant compared to the wild-type $M A T-1$, and 391 DEGs in non-mating (Ss $\triangle M A T-1 b$ X MAT-2) mixture compared to mating (MAT-1 X MAT-2) mixture. Among them, 28 annotated genes (12 up-regulated and 16 down-regulated, Table 3) were common in these two sets of comparisons, thus are most likely associated with mating/filamentous growth and subject to regulation by bE/bW heterodimeric transcription factor.

In the enriched KEGG pathway, we noticed that sucrose/starch metabolism pathway was altered in the Ss $\triangle M A T-1 b$ mutant in a way that intracellular glucose is predicted to be reduced and 1,3 $\beta$-glucan elevated. Also, glucoamylase encoded gene was identified as potentially b-locus induced (Table 3). Our results (Fig. 3b) showed in contrast to our prediction, that elevated glucose level repressed, but not promoted, filamentous growth and/or mating. We infer that the timing ( $24 \mathrm{~h}$ post mating) for detecting glucoamylase transcription might not be suitable, when at this time point the transcripts started translating into proteins. Therefore, the apparent low level of glucoamylase in non-mating sets would reflect active glucose production, and b-locus may actually repress glucoamylase during mating and/or filamentous growth. We further hypothesize that glucose may be channeled to synthesis of 1,3- $\beta$-glucan during S. scitamineum filamentous growth after mating and likely regulated by b-locus, through repression of glucoamylase. As $1,3-\beta$ glucan is an anti-oxidant, its production may relief the cell from endogenous oxidative stress therefore Hog1 was not induced in wild-type condition. In b-deletion condition, glucose level may elevated and therefore repress filamentous growth; meanwhile the reduced1,3- $\beta$-glucan level resulted in endogenous oxidative stress and induction of Hog1 as a response. Ss $\triangle M A T-1 b$ mutant was slightly more resistant to $\mathrm{H} 2 \mathrm{O} 2$, likely due to hyper-induced Hog1. Our hypothesis was supported by the observation that glucosedepleted medium (SC) promoted filamentous growth in the mating mixture of MAT-1 X MAT-2 spores (Fig. 3c). It has been reported that glucose plays an important role in asexual/sexual sporulation in other pathogenic/filamentous fungi, including Magnaporthe oryzae [35], U. maydis [36], and Fusarium graminearum [37]. Also, glucose was reported to suppress mating competency in Candida albicans [16]. Our results fit well with the established notion that glucose promotes unicellular spore/cell production while represses filamentous growth, thus acting as a switch between dimorphic transition.

Another interesting observation from common DEGs and KEGG enrichment is that the stress-activating MAPK signaling pathway mediated by Hog1 was significantly upregulated, in both $S s \triangle M A T-1 b$ mutant and Ss $\triangle M A T-1 b$ X MAT-2 mixture. One possibility is that, elevated glucose production in Ss $\triangle M A T-1 b$ haploid resulted in reduced 
production of $1,3-\beta$-glucan, which is also known as an anti-oxidant. As a result, HOG1 was transcriptionally induced in response to elevated intracellular oxidative level. Alternatively, HOG1 may be repressed by the bE/bW transcriptional complex, directly or indirectly, during mating. Our tests showed that $S s \triangle M A T-1 b$ is less sensitive to oxidative stress. Meanwhile, repression on colonial growth caused by anti-oxidant GSH was more prominent with presence of glucose. Overall, these results suggest that Hog1 MAPK signaling may be repressed by the bE/bW transcriptional complex. Such observation is consistent with the reported function of the Hog1 ortholog in Candida albicans that negatively regulates its mating capacity [16]. However, we are not aware of whether SsHOG1 is one of the direct targets (class I) genes of the bE/bW transcriptional complex, or among the indirect (class II) targets, as no obvious $b$-locus binding site (bbs $[38,39]$ ) was predicted in the promoter region of SsHOG1.

It has been reported in $U$. maydis that $\mathrm{GO}$ categories "Cell Cycle", "Chromosome" and "DNA metabolic process" were significantly enriched as b-down-regulated genes [40]. However, we observed that "DNA replication" was enriched as up-regulated GO terms in nonmating mixture (Fig. 2; Additional file 5: Table S5; GO: 0006260), and mitosis regulator Cek1 [24] and GAP Bem2 that related to mitosis [29] were up-regulated in non-mating sets, which may also account for the failure of mating, with deletion of $b$-locus in MAT-1.

\section{Conclusions}

Overall, our transcriptome analysis contributes to prediction of candidate genes of the regulatory cascade of $S$. scitamineum $b$-locus, in terms of mating and/or filamentous growth after recognition of opposite sex mediated by the $a$-locus. In future, further investigation on such candidate genes would help elucidate molecular mechanism of S. scitamineum mating, including but not limited to, $b$-locus regulating cell fate decision, morphogenesis, carbon/nitrogen metabolism, mitosis, stress (oxidative) response, etc. This would certainly enrich our knowledge in fungal sexual differentiation and/or pathogenesis, and likely of great potential towards development/design of anti-fungal pathogen strategy.

\section{Ethics and consent to participate}

Not applicable.

\section{Consent to publish}

Not applicable.

\section{Availability of data and materials}

All the data supporting our findings is contained within the manuscript and supplementary files.

\section{Additional files}

Additional file 1: Table S1. List of All-Unigene De novo assembled in this study. (XLS $2360 \mathrm{~kb}$ )

Additional file 2: Table S2. List of DEGs $(p \leq 0.05)$ in the Ss $\triangle M A T-1 b$ mutant compared to the wild-type MAT-1. (XLS $396 \mathrm{~kb}$ )

Additional file 3: Table S3. List of DEGs $(p \leq 0.05)$ in non-mating mixture of Ss $\triangle M A T-16$ X MAT-2, compared to the mating mixture of MAT-1 X MAT-2 (XLS $185 \mathrm{~kb}$ )

Additional file 4: Table S4. List enriched GO in Ss $\triangle M A T-16$ mutant compared to the wild-type MAT-1. (XLS $41 \mathrm{~kb})$

Additional file 5: Table S5. List enriched $\mathrm{GO}$ in non-mating mixture of Ss $\triangle M A T-16$ X MAT-2, compared to the mating mixture of MAT-1 X MAT-2 (XLS $42 \mathrm{~kb})$

Additional file 6: Table S6. List of enriched KEGG terms in SS $\triangle M A T-1 b$ mutant compared to the wild-type MAT-1. (XLS $47 \mathrm{~kb}$ )

Additional file 7: Table S7. List of enriched KEGG terms in non-mating mixture of Ss $\triangle M A T-16 \times$ MAT-2, compared to the mating mixture of MAT-1 X MAT-2. (XLS $48 \mathrm{~kb})$

Additional file 8: Figure S1. Starch/sucrose metabolism pathway (ko00500) common in both haploid and mating sets. Red box denotes up-regulated genes, and green box are down-regulated genes. The source of image is from KEGG pathway database (http://www.kegg.jp/) developed by Kanehisa Laboratories, and is allowed to reproduced for academic purpose. (TIF $1572 \mathrm{~kb}$ )

Additional file 9: Figure S2. MAPK signaling pathway (ko04010) common in both haploid and mating sets. Red box denotes up-regulated genes, and green box are down-regulated genes. The source of image is from KEGG pathway database (http://www.kegg.jp/), developed by Kanehisa Laboratories, and is allowed to reproduced for academic purpose. (TIF $1373 \mathrm{~kb}$ )

\section{Competing interests}

The authors declare that they have no competing interests.

Authors' contributions

MY and EC performed the majority of the experiments; WD performed data analysis of transcriptome sequencing; CC and YZD analyzed the data; ZJ and LHZ jointly designed the experiments; YZD and LHZ drafted and edited the manuscript. All authors have read and approved the final version of the manuscript.

\section{Acknowledgements}

We thank OEbiotech.co.Itd for technical support in our transcriptome analysis.

\section{Funding}

This research was funded by the National 973 Program of China (No.2015CB150600) and the Innovative R\&D Team Program of Guangdong Province, China (No. 2013S034).

\section{Author details}

${ }^{1}$ Guangdong Province Key Laboratory of Microbial Signals and Disease Control, College of Agriculture, South China Agricultural University,

Guangzhou, Peoples' Republic of China. ${ }^{2}$ Guangdong Innovative and Entepreneurial Research Team of Sociomicrobiology Basic Science and Frontier Technology, South China Agricultural University, Guangzhou, Peoples' Republic of China. ${ }^{3}$ Integrative Microbiology Research Centre, South China Agricultural University, Guangzhou, Peoples' Republic of China.

Received: 27 January 2016 Accepted: 5 May 2016

Published online: 16 May 2016

References

1. Singh N, Somai BM, Pillay D. Smut disease assessment by PCR and microscopy in inoculated tissue cultured sugarcane cultivars. Plant Sci. 2004;167(5):987-94. 
2. Que Y, Xu L, Wu Q, Liu Y, Ling H, Liu Y, Zhang Y, Guo J, Su Y, Chen J, et al. Genome sequencing of Sporisorium scitamineum provides insights into the pathogenic mechanisms of sugarcane smut. BMC Genomics. 2014;15:996.

3. Albert HH, Schenck S. PCR amplification from a homolog of the bE matingtype gene as a sensitive assay for the presence of U. scitaminea DNA. Plant Dis. 1996:80:1189-92.

4. Yoder OC, Weltring K, Turgeon BG, Garber RC, VanEtten HD. Technology for molecular cloning of fungal virulence genes. Biol Mol Biol Plant Pathogen Interact. 1986;1:371-84.

5. Vollmeister E, Schipper K, Baumann S, Haag C, Pohlmann T, Stock J, Feldbrugge M. Fungal development of the plant pathogen Ustilago maydis. FEMS Microbiol Rev. 2012;36(1):59-77.

6. Wahl R, Zahiri A, Kamper J. The Ustilago maydis b mating type locus controls hyphal proliferation and expression of secreted virulence factors in planta. Mol Microbiol. 2010;75(1):208-20.

7. Fedler M, Luh KS, Stelter K, Nieto-Jacobo F, Basse CW. The a2 mating-type locus genes lga2 and rga2 direct uniparental mitochondrial DNA (mtDNA) inheritance and constrain mtDNA recombination during sexual development of Ustilago maydis. Genetics. 2009;181(3):847-60.

8. Bölker M, Genin S, Lehmler C, Kahmann R. Genetic regulation of mating and dimorphism in Ustilago maydis. Can J Bot. 1995:73(S1):320-5.

9. Gillissen B, Bergemann J, Sandmann C, Schroeer B, Bolker M, Kahmann R. A two-component regulatory system for self/non-self recognition in Ustilago maydis. Cell. 1992;68(4):647-57.

10. Kamper J, Reichmann M, Romeis T, Bolker M, Kahmann R. Multiallelic recognition: nonself-dependent dimerization of the bE and bW homeodomain proteins in Ustilago maydis. Cell. 1995;81(1):73-83.

11. Feldbrugge M, Kamper J, Steinberg G, Kahmann R. Regulation of mating and pathogenic development in Ustilago maydis. Curr Opin Microbiol. 2004; 7(6):666-72.

12. Taniguti LM, Schaker PD, Benevenuto J, Peters LP, Carvalho G, Palhares A, Quecine MC, Nunes FR, Kmit MC, Wai A, et al. Complete Genome Sequence of Sporisorium scitamineum and Biotrophic Interaction Transcriptome with Sugarcane. PLoS One. 2015;10(6):e0129318.

13. Sun L, Yan M, Ding Z, Liu Y, Du M, Xi P, Liao J, Ji L, Jiang Z. Improved dominant selection markers and co-culturing conditions for efficient Agrobacterium tumefaciens-mediated transformation of Ustilago scitaminea. Biotechnol Lett. 2014;36(6):1309-14

14. Yan M, Zhu G, Lin S, Xian X, Chang C, Xi P, Shen W, Huang W, Cai E, Jiang $Z$, et al. The mating-type locus $b$ of the sugarcane smut Sporisorium scitamineum is essential for mating, filamentous growth and pathogenicity. Fungal Genet Biol. 2016;86:1-8.

15. Yan M, Zhu G, Lin S, Xian X, Chang C, Xi P, Shen W, Huang W, Cai E, Jiang $Z$, et al. The Mating Type Gene b Locus of Sugarcane Smut Sporisorium scitamineum Is Essential for Mating, Filamentous Growth and Pathogenicity. Fungal Genet Biol. 2015;86:1-8.

16. Liang $\mathrm{SH}$, Cheng $\mathrm{H}$, Deng FS, Tsai PA, Lin CH. A novel function for Hog1 stress-activated protein kinase in controlling white-opaque switching and mating in Candida albicans. Eukaryot Cell. 2014;13(12):1557-66.

17. Hansen KD, Brenner SE, Dudoit S. Biases in Illumina transcriptome sequencing caused by random hexamer priming. Nucleic Acids Res. 2010;38(12):e131

18. Trapnell C, Pachter L, Salzberg SL. TopHat: discovering splice junctions with RNA-Seq. Bioinformatics. 2009;25(9):1105-11.

19. Langmead B, Salzberg SL. Fast gapped-read alignment with Bowtie 2. Nat Methods. 2012;9(4):357-9.

20. Florea L, Song L, Salzberg SL. Thousands of exon skipping events differentiate among splicing patterns in sixteen human tissues. F1000 Research. 2013;2:188.

21. Kanehisa M, Araki M, Goto S, Hattori M, Hirakawa M, Itoh M, Katayama T, Kawashima S, Okuda S, Tokimatsu T, et al. KEGG for linking genomes to life and the environment. Nucleic Acids Res. 2008;36(Database issue):D480-484.

22. Altschul SF, Gish W, Miller W, Myers EW, Lipman DJ. Basic local alignment search tool. J Mol Biol. 1990;215(3):403-10.

23. Abdi H. Bonferroni and idák corrections for multiple comparisons. In: Salkind NJ, editor. Encyclopedia of measurement and statistics. Thousand Oaks: Sage; 2007.

24. Samejima I, Yanagida M. Identification of cut8+ and cek1+, a novel protein kinase gene, which complement a fission yeast mutation that blocks anaphase. Mol Cell Biol. 1994;14(9):6361-71.

25. Li R, Puri S, Tati S, Cullen PJ, Edgerton M. Candida albicans Cek1 mitogenactivated protein kinase signaling enhances fungicidal activity of salivary histatin 5. Antimicrob Agents Chemother. 2015;59(6):3460-8.
26. Bahn YS, Geunes-Boyer S, Heitman J. Ssk2 mitogen-activated protein kinase kinase kinase governs divergent patterns of the stress-activated Hog1 signaling pathway in Cryptococcus neoformans. Eukaryot Cell. 2007:6(12):2278-89.

27. Zapater M, Sohrmann M, Peter M, Posas F, de Nadal E. Selective requirement for SAGA in Hog 1-mediated gene expression depending on the severity of the external osmostress conditions. Mol Cell Biol. 2007;27(11):3900-10.

28. Van Wuytswinkel O, Reiser V, Siderius M, Kelders MC, Ammerer G, Ruis H, Mager WH. Response of Saccharomyces cerevisiae to severe osmotic stress: evidence for a novel activation mechanism of the HOG MAP kinase pathway. Mol Microbiol. 2000;37(2):382-97.

29. Kim YJ, Francisco L, Chen GC, Marcotte E, Chan CS. Control of cellular morphogenesis by the Ip12/Bem2 GTPase-activating protein: possible role of protein phosphorylation. J Cell Biol. 1994;127(5):1381-94.

30. Attwood PV. Histidine kinases from bacteria to humans. Biochem Soc Trans. 2013;41(4):1023-8

31. Riechmann JL, Heard J, Martin G, Reuber L, Jiang C, Keddie J, Adam L, Pineda O, Ratcliffe OJ, Samaha RR, et al. Arabidopsis transcription factors: genome-wide comparative analysis among eukaryotes. Science. 2000; 290(5499):2105-10.

32. Huang D, Sumegi J, Dal Cin P, Reith JD, Yasuda T, Nelson M, Muirhead D, Bridge JA. C11 orf95-MKL2 is the resulting fusion oncogene of $t(11 ; 16)(q 13 ; p 13)$ in chondroid lipoma. Genes Chromosomes Cancer. 2010;49(9):810-8.

33. Binder G. Short stature due to SHOX deficiency: genotype, phenotype, and therapy. Horm Res Paediatr. 2011;75(2):81-9.

34. Ashburner M, Lewis S. On ontologies for biologists: the Gene Ontologyuntangling the web. Novartis Found Symp. 2002;247:66-80. discussion 80-63, 84-90, 244-252.

35. Deng YZ, Ramos-Pamplona M, Naqvi NI. Autophagy-assisted glycogen catabolism regulates asexual differentiation in Magnaporthe oryzae. Autophagy. 2009;5(1):33-43.

36. Nadal M, Gold SE. The autophagy genes ATG8 and ATG1 affect morphogenesis and pathogenicity in Ustilago maydis. Mol Plant Pathol. 2010;11(4):463-78

37. Josefsen L, Droce A, Sondergaard TE, Sorensen JL, Bormann J, Schafer W, Giese $\mathrm{H}$, Olsson S. Autophagy provides nutrients for nonassimilating fungal structures and is necessary for plant colonization but not for infection in the necrotrophic plant pathogen Fusarium graminearum. Autophagy. 2012;8(3):326-37.

38. Romeis T, Brachmann A, Kahmann R, Kamper J. Identification of a target gene for the bE-bW homeodomain protein complex in Ustilago maydis. Mol Microbiol. 2000:37(1):54-66.

39. Brachmann A, Weinzierl G, Kamper J, Kahmann R. Identification of genes in the bW/bE regulatory cascade in Ustilago maydis. Mol Microbiol. 2001;42(4):1047-63.

40. Heimel $K$, Scherer M, Vranes $M$, Wahl R, Pothiratana C, Schuler D, Vincon V, Finkernagel F, Flor-Parra I, Kamper J. The transcription factor Rbf1 is the master regulator for b-mating type controlled pathogenic development in Ustilago maydis. PLoS Pathog. 2010;6(8):e1001035.

\section{Submit your next manuscript to BioMed Central and we will help you at every step:}

- We accept pre-submission inquiries

- Our selector tool helps you to find the most relevant journal

- We provide round the clock customer support

- Convenient online submission

- Thorough peer review

- Inclusion in PubMed and all major indexing services

- Maximum visibility for your research

Submit your manuscript at www.biomedcentral.com/submit 